\title{
Design and fabrication of unsteady electrokinetic microactuator arrays for turbulent boundary layer control
}

\author{
Francisco J Diez and Werner J A Dahm \\ Laboratory for Turbulence and Combustion (LTC), Department of Aerospace Engineering, \\ The University of Michigan, Ann Arbor, MI 48109-2140, USA
}

Received 12 January 2004

Published 13 July 2004

Online at stacks.iop.org/JMM/14/1307

doi:10.1088/0960-1317/14/10/004

\begin{abstract}
The development of a micro-electro-kinetic-actuator (MEKA) array for the study and control of the viscous sublayer of a turbulent boundary layer is presented. Several generations of such MEKA arrays have been fabricated that explore the use of non-conventional MEMS materials such as quartz, polymers or mylar and are combined where needed with conventional MEMS design and fabrication processes. The present study is the first attempt to exploit the potential advantages of using the electrokinetic principle as the basis for a new class of microscale actuators suitable for active sublayer control. Among such advantages are that these actuators have no moving parts, and that they achieve the flow rates required for this type of flow control. Moreover, the inherent problem of matching the length and time scales between microactuators and the physical system being controlled makes the viscous sublayer a natural choice for these types of actuators. The electrokinetic drivers are fabricated inside microchannels, $250 \mathrm{~mm}$ to $2 \mathrm{~mm}$ in diameter, using a liquid-phase polymerization process that generates $1 \mu \mathrm{m}$ doped pores. This process greatly simplifies the fabrication of a large number of actuators, and using this technique we are able to fill 100\% of the 25600 microchannels that form the typical MEKA 5 array. The array is fabricated using a novel three-layer design that contains (i) a top layer with the actuator nozzles, electrodes and leadouts, (ii) a center layer containing the individually addressable electrokinetic driver channels in which electrolyte pumping occurs in response to a time-varying electric field that will induce volume displacement in the sublayer and (iii) a bottom layer containing an electrolyte reservoir and common electrode. The functionality incorporated in this three-layer design with independent unit cells demonstrate all the elements needed for turbulent boundary layer control.
\end{abstract}

\section{Introduction}

The development of robust, survivable, long-lifetime, highbandwidth, high-authority, low-power fluid microactuators currently represents the single greatest need in the application of MEMS technologies to the control of vehicles, as well as to a wide range of other areas. Active sublayer control for drag reduction in turbulent boundary layers is one of the highest-impact applications for 'smart' control of turbulence.
A reduction of just 1-2\% in the drag created by the turbulent boundary layer on a commercial airplane or ship can translate into large reductions in fuel consumption and operating costs. Similarly, flow-control devices capable of on-demand vortex generation over delta wings, strakes and other control surfaces on fighter aircraft would permit dramatic increases in maneuverability, and large reductions in radar crosssection through elimination of traditional control surfaces. Such benefits of microsystems-based flow control extend to 
naval surface and undersea vehicles as well, including ships, submarines and torpedoes, as well as to unmanned vehicles used in a variety of applications such as UAV, UCAV and MAV. Similar technologies could also be applied to supersonic vehicles for aerodynamic noise reduction, and would find use in a wide range of flow control situations involved in propulsion systems, spanning from control of mixing in advanced gas turbine combustors, to active control of surge and stall in compressors.

The thin boundary layer that exists directly adjacent to the surface of any practical vehicle is one of the most powerful nonlinear systems found in nature, capable of amplifying the effect of small microactuator-induced perturbations into very large changes in the aerodynamic forces and moments acting on the vehicle. Moreover, the inherent problem of matching the length and time scales between microactuators and the physical system being controlled makes the viscous sublayer of a turbulent boundary layer a natural choice for microsystemsbased control. The exceedingly small length scales associated with flow structures that are naturally present in the viscous sublayer of turbulent boundary layers are ideally matched to microscale actuators. However the high performance required for sublayer control places rather stringent limits on the types of actuators that may be suitable for this task.

Recent experimental and numerical studies specifically related to the development of microactuators for control of near-wall turbulence have been reviewed by Moin and Bewley [1], Gad-el-Hak [2, 3], McMichael [4], Ho and Tai [5], Pollard [6], Lumley and Blossey [7], Löfdahl and Gad-el-Hak [8] and Bewley [9], among others. In particular, Ho and Tai $[5,6]$ and Tsao et al [10] demonstrated an electromagnetically activated microflap actuator that could be deflected to produce an upward velocity in opposition to the wall-normal velocity produced by the streamwise sublayer vortices, but to date this approach has not been applied to demonstrate drag reduction in turbulent boundary layers. Other investigations have examined the use of piezoelectrically driven actuators to control streamwise vortices in turbulent boundary layers (Jacobson and Reynolds [11, 12], Rathnasingham and Breuer [13]). The latter study achieved a maximum reduction of $31 \%$ in $u_{\mathrm{rms}}$ in a low Reynolds number turbulent boundary layer test. Other attempts to control streamwise vortices include the selective-suction actuators of Myose and Blackwelder [14]. Direct numerical simulations of turbulent channel flows using a similar actuation methodology, based on local suction and blowing at the wall, was performed by Choi et al $[15,16]$ yielding 20-30\% reduction in skin friction. Similar numerical experiments have been conducted by Hammond et al [17] and Rebbeck and Choi [18].

The central technology required to achieve practical vehicle control rests largely in the development of robust, highperformance, highly survivable, low-power microactuators for integration in large, dense arrays that can meet the realistic operating requirements noted earlier. The present study (Diez-Garias et al [19]) is the first to examine the electrokinetic principle as the basis for a new class of microactuator arrays that are potentially suitable for active sublayer control on real aeronautical and hydronautical vehicles under practical conditions. This paper does not aim to reveal new results relevant to elementary electrochemical processes in electroosmotic pumps. Instead, it uses existing electrokinetic pump technology to design and fabricate the first large, dense arrays of electrokinetic microactuators for sublayer control of turbulent boundary layers. A complete system architecture for such microactuator arrays has also been developed as part of this study. This novel system architecture is based on groups of actuators within a local 'unit cell'. The architecture further permits a greatly simplified control-logic approach, also developed as part of this study [19,20], that is based on a simple look-up table resident in firmware within each unit cell.

\section{Microactuator performance requirements}

Turbulent boundary layers obey a universal structure and scaling under so-called 'equilibrium' conditions, which typically apply over the vast majority of a vehicle surface. It is necessary to be aware of at least the basics of this general structure and its scaling in order to understand the approach being taken here, and to understand the performance requirements that they dictate for microactuator arrays. The central issue concerns the form of the mean velocity profile $u(y)$ with increasing distance $y$ from the vehicle surface (referred to generically as the 'wall', located at $y \equiv 0$ ).

The boundary layer has a two-layer structure-an 'inner layer' directly adjacent to the wall within which molecular transport dominates, and an 'outer layer' within which turbulent transport dominates. Within the inner layer, owing to the dominance of diffusion, the momentum flux density must be roughly constant at a value called the 'wall shear stress', $\tau_{\mathrm{w}}$, literally the value of the shear stress at the wall. All processes within this layer scale with this wall shear stress, the viscosity ' $v$ ', the fluid density $\rho$ and the distance $y$ above the wall. Such near-wall scalings are thus expressed in terms of a characteristic velocity $u_{\tau} \equiv\left(\tau_{\mathrm{w}} / \rho\right)^{1 / 2}$, and a characteristic length $l_{\tau} \equiv v / u_{\tau}$ often termed the 'wall unit' [21]. The scaled velocity profile $u^{+}\left(y^{+}\right)$, where $u^{+} \equiv u / u_{\tau}$ and $y^{+} \equiv y / l_{\tau}$, is then universal throughout the inner layer for all equilibrium turbulent boundary layers [21].

Streamwise vortical structures form naturally with a spacing, in the scaled spanwise coordinate $z^{+} \equiv z / l_{\tau}$, of about $z^{+} \approx 100$. The length of these 'sublayer vortices' is typically about $x^{+} \approx 1000$, but varies from about 400 to 1500 . In other words, the vortices are about 1000 wall units $\left(l_{\tau}\right)$ long, and are spaced about 100 wall units apart. Since the vortices are located roughly 10 wall units above the wall, they will advect at a speed $u^{+} \approx 10$, and thus move over any fixed point on the wall with a frequency of about $f^{+} \approx 10^{-2}$. Further details can be found in Kline et al [22], Kim et al [23] and Robinson [24]. Table 1 shows a list of full-scale aeronautical and hydronautical vehicles, giving overall vehicle length $L$ and speed $U$ used to obtain their characteristic $l_{\tau}$ and $f_{\tau}$.

These alternating pairs of counter-rotating streamwise vortical structures at the upper edge of the viscous sublayer are responsible for the 'bursting' process in which relatively large amounts of low-speed (high-speed) fluid, in a wallfixed frame, are suddenly ejected (sweeped) from (into) the near-wall region. Interrupting the sweep and ejection events involved in this bursting process has long been an objective in fluid mechanics and aerodynamics. Laboratory 
Table 1. Full-scale aeronautical and hydronautical vehicle types considered in section 2 , giving overall vehicle length $L$ and speed $U$ used to obtain viscous sublayer characteristics for various values of the pressure gradient parameter II at locations $x / L=0.25,0.50,0.75$, and 1.00 . The resulting $l_{\tau}$ and $f_{\tau}$ values (where $l^{+}=10$ and $f^{+}=10^{-2}$ ) determine the required performance characteristics shown in figure 2 for active sublayer control on each vehicle type at full-scale operating conditions. Note that the nozzle $u^{+}<0.1$ in all cases.

\begin{tabular}{lclll}
\hline Vehicle class & Length $(\mathrm{m})$ & Speed $\left(\mathrm{m} \mathrm{s}^{-1}\right)$ & $l_{\tau}(\mu \mathrm{m})$ & $f_{\tau}(\mathrm{kHz})$ \\
\hline Transport aircraft (wing) & 3 & 200 & $1.8-3.1$ & $15-41$ \\
Supersonic fighter (wing) & 5 & 300 & $1.3-2.1$ & $30-80$ \\
UAV (wing) & 1 & 30 & $25-54$ & $0.05-0.22$ \\
Naval surface ship (hull) & 180 & 30 & $3.6-5.0$ & $0.41-0.84$ \\
Submarine (hull) & 170 & 10 & $2.9-4.2$ & $0.5-1.2$ \\
Torpedo & 5 & 10 & $2.7-4.5$ & $0.5-1.4$ \\
Automotive (body) & 2 & 30 & $10-19$ & $0.4-1.4$ \\
\hline
\end{tabular}

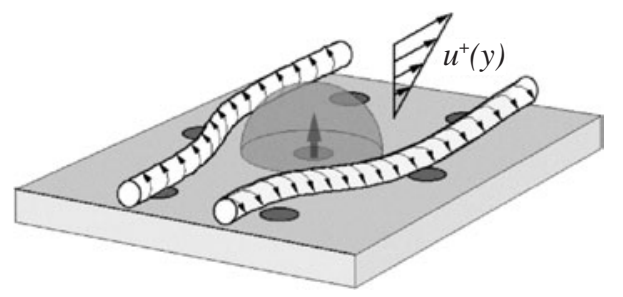

Figure 1. Idealized notional representation showing the lateral displacement of streamwise sublayer vortices induced by volumetric pumping from an actuator. Note that the Reynolds number $R e$ associated with the flow issuing from the actuator must be $O(1)$ or smaller, so that the actuator flow serves as a point volume source to provide effective lateral displacement. At larger $R e$ values, the flow issuing from the actuator would fundamentally change to become jet-like, and would then no longer induce effective lateral displacements.

experiments and numerical simulations have shown that this can be accomplished by acting on the streamwise vortices in any of a number of ways so as to offset the Biot-Savart-induced velocities that lead to the sudden bursting. For the present microactuator arrays, the individual actuators serve as point volume sources that displace (either positively or negatively) a fixed volume of fluid between the wall and the inner layer as shown schematically in figure 1 . The motion that this volume source field induces serves to displace the streamwise vortices along the spanwise direction, thereby locally increasing or decreasing the separation between the two elongated vortices in a streamwise vortex pair. This counteracts the Biot-Savartinduced motion of the vortices on each other, thus interrupting the bursting process.

Key performance requirements involve the microactuator spacing, frequency and flow rate needed to achieve adequate displacement of individual sublayer vortical structures. The latter has been studied in more detail by Diez-Garias [20] and Diez and Dahm [25]. From the forgoing summary of the sublayer vortex structure and dynamics, it is apparent that such actuators must be separated by typically 100 wall units, and displace a volume of fluid with an equivalent hemispherical radius of the order of 10 wall units $1_{\tau}$, with a step response that corresponds to a scaled frequency of $f^{+} \approx 10^{-2}$. The resulting exit velocity varies greatly from one application to the next, but there is no direct requirement on the nozzle $u^{+}$, though the nozzle Reynolds number must not exceed $0(1)$. Accordingly, figure 2 shows the results obtained for the required point microactuator spacing and frequency response at four downstream locations $(x / L=0.25,0.5,0.75$ and 1.0$)$

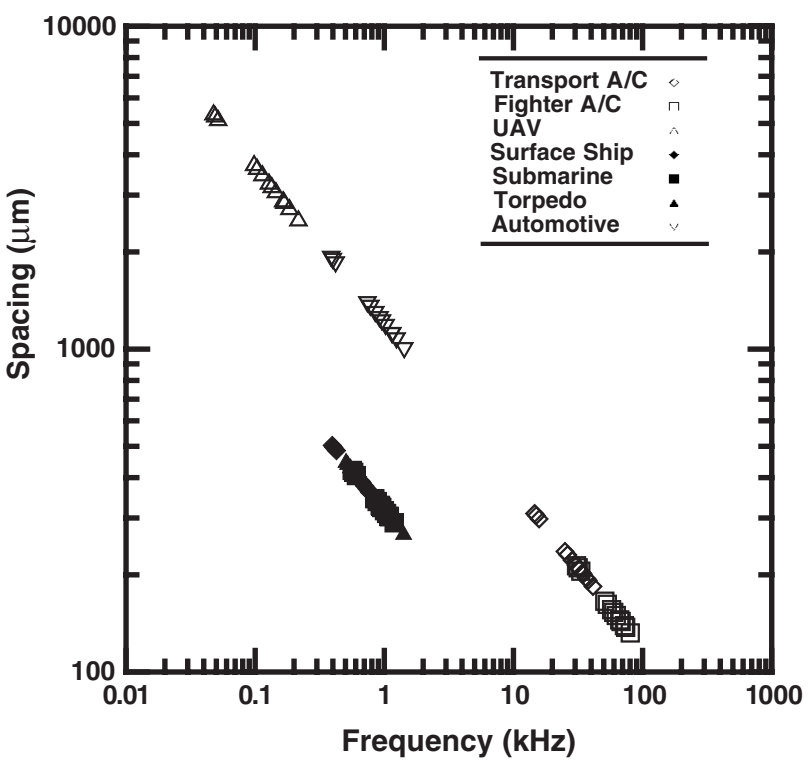

Figure 2. Microactuator spacing and frequency requirements for active sublayer control by manipulation of streamwise vortical structures, showing results for aeronautical and hydronautical vehicle types listed in table 1 . Note that hydronautical applications all require microactuator arrays with approximately $300 \mu \mathrm{m}$ actuator spacings and $1 \mathrm{kHz}$ frequency response.

and for four different pressure gradients. These requirements are relatively benign for the UAV application, where actuator spacings of several millimeters and step response of $100 \mathrm{~Hz}$ are sufficient to act on virtually every sublayer streak. At the other extreme, the supersonic fighter and transport aircraft require actuator spacings of 100-200 $\mu \mathrm{m}$ and step response of $10-90 \mathrm{kHz}$. The various hydronautical applications require microactuator spacings roughly $300 \mu \mathrm{m}$ but frequencies of only about $1 \mathrm{kHz}$.

\section{System architecture for microactuator arrays}

The small actuator spacing required for sublayer control on real vehicles, as seen in figure 2 , implies that large and dense arrays of microactuators must be used to cover key parts of the vehicle surface. However, the inherently local nature of the sublayer vortex dynamics and bursting process suggests that such large arrays can be composed of tiles and these of much smaller independent 'unit cells', each with their own sensors, control processing and actuators. This highly local 
nature of the problem thus permits a system architecture for such microactuator arrays that greatly simplifies the sublayer control of turbulent boundary layers. A complete description of this architecture can be found in Diez-Garias [20] and Diez and Dahm [25] and is insensitive to the alignment of the streamwise vortices relative to the array. Each unit cell in this architecture is composed of a collocated array of wall shear stress sensors, electrokinetic microactuators and local digital signal processing (DSP) capability. This unit-cell concept is sufficiently simple to realistically permit integration of the present electrokinetic microactuators into a complete system for active sublayer control on full-scale vehicles under realistic operating conditions.

Each unit cell contains a $4 \times 4$ array of electrokinetic actuators that provide the volume displacements within the near-wall region of the turbulent boundary layer to laterally displace the streamwise sublayer vortices. Although the fundamental unit-cell architecture can accommodate essentially any type of actuator, the electrokinetic microactuators that are the principle focus of this study offer several benefits that make them potentially well suited for active sublayer control. The electrokinetic principle on which these microactuators function (see section 4) requires no moving parts, with the volumetric pumping being induced by an impulsively applied electric field. As a result, these electrokinetic microactuators can be significantly more robust than many other actuators based on moving parts. Moreover, the scalings that govern the electrokinetic effect provide significant performance advantage for actuators fabricated at the microscale. In particular, such electrokinetic microactuators can achieve an extremely high impulse response, and equivalent steady flow rates sufficient to meet the requirements noted in section 2 for active sublayer control under full-scale vehicle operating conditions. Such microactuators can also be readily sized to fit with the unitcell architecture, and do not involve any parts that protrude into the flow.

Furthermore, such electrokinetic microactuators can be fabricated with a comparatively simple three-layer design, as indicated in figure 3. This three-layer design uses a center layer containing individual electrokinetic driver channels formed in an appropriate substrate material and filled with a porous polymer structure. This structure can readily be wetted with electrolyte by simple capillary wicking action and the double layer forms (see section 4) within the pores (channels) in the porous polymer. The electrokinetic flow induced in the electrolyte (inside the porous polymer) when an electric field is applied across any individual center-layer channel serves to displace the working fluid from the top layer. This top layer contains individual actuator nozzles and electrodes together with leadouts to the unit-cell DSP. The bottom layer serves as a common electrolyte reservoir for the unit cell (or, alternatively, for the entire tile). Although not implemented here, a thin and flexible membrane could be used to separate the working fluid from the electrolyte to prevent any leakage. Nevertheless, since the leakage would be small, electrolyte could be added to a main reservoir from time to time to account for any lost due to leakage.

Power for the electrokinetic microactuators is distributed throughout the tile via a simple bus structure composed of

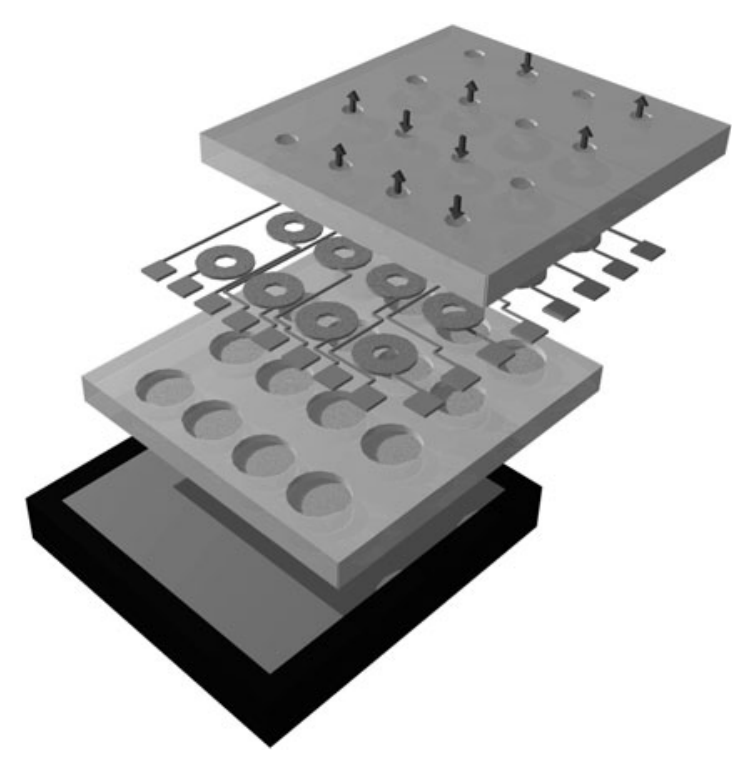

Figure 3. Fundamental three-layer design of electrokinetic microactuator arrays at the unit-cell level, showing a top layer containing individual actuator nozzles, electrodes and leadouts, a center layer containing the electrokinetic driver channels in which electrolyte pumping occurs in response to an applied electric field, and an electrolyte reservoir and common electrode in the bottom layer.

parallel horizontal and vertical line conductors in the top layer across the entire tile. The common electrolyte reservoir serves as the other electrode. The high level of redundancy provided by the top-layer bus structure ensures continuity of a conductive path to all actuators on the tile even in the event of a local damage site on the tile or a local failure along any of the line conductors. A set of leadouts run within the top layer from each individual actuator electrode to a terminal located near one of the common power bus lines. On each clock cycle, the DSP selectively connects each electrode terminal to the power bus, with either positive or negative polarity, to provide for positive or negative volume displacement by each individual actuator, or else leaves this connection open to provide for zero displacement by the actuator.

\section{Unsteady electrokinetic pumping}

\subsection{Quasy-steady EK pumping}

The electrokinetic microactuators that are the subject of this work function on the basis of the electrokinetic effect shown schematically in figure 4 (e.g., Potter [26], Burgreen and Nakache [27]) and it was first noted by Reuss (1809) [28]. While this effect inherently operates at the microscale, it is widely used in a variety of practical devices and processes to produce macroscale effects. Examples include transport processes in emulsions and suspensions, as well as soil remediation processes, to name just a few. The present study is the first attempt to exploit its potential advantages as the basis for a new class of microscale actuators suitable for active sublayer control in turbulent boundary layers at fullscale vehicle operating conditions.

The Navier-Stokes equation can be used to obtain the detailed outer-layer structure and the resulting electrokinetic 


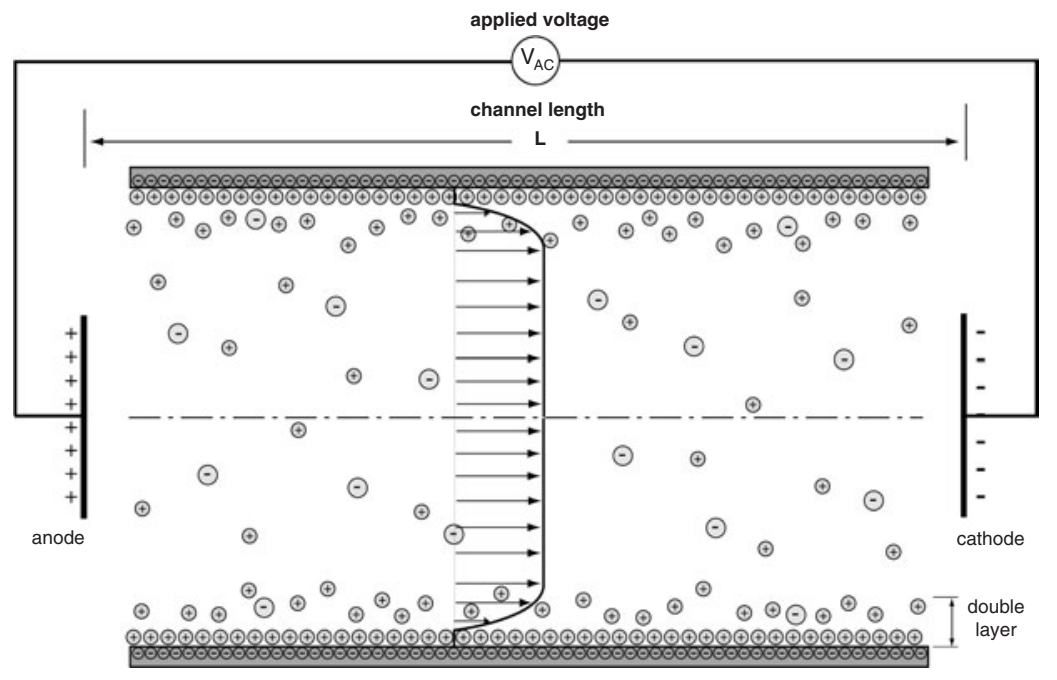

Figure 4. Schematic showing electroosmotic flow induced by an applied electric field along a pore or capillary channel. The electric field induces drift in the mobile ions within the diffuse outer layer, which then collisionally transfer momentum to the electrolyte. Subsequent collisional transport (diffusion) transfers this motion throughout the channel.

pumping that it induces under the influence of an applied electric field (e.g., Burgreen and Nakache [27], and Rice and Whitehead [29]). Simplifications to the NavierStokes equation given by Rice and Whitehead [29] for the electrokinetic flow in a narrow cylindrical capillary of radius $w$ gives the following equation of motion:

$$
\mu \frac{1}{r} \frac{\mathrm{d}}{\mathrm{d} r}\left(r \frac{\mathrm{d} V_{z}}{\mathrm{~d} r}\right)=-P_{z}-E_{z}\left(\varepsilon \kappa^{2} \zeta \frac{I_{\mathrm{o}}(\kappa r)}{I_{\mathrm{o}}(\kappa w)}\right) .
$$

where $\varepsilon$ is the dielectric constant, $\kappa$ is the Debye length, $I_{\mathrm{o}}$ is the zero-order modified Bessel function of the first kind, $P_{z}$ is the pressure gradient, $E_{z}$ is an axial electric field and the wall of the capillary is at a potential $\zeta$.

For the present electrokinetic microactuators, no pressure gradient is applied, and the flow instead results entirely from the applied electric field $E_{\mathrm{z}}$. Solving (1) under these conditions, the velocity $v_{z}$ is obtained as

$$
v_{z}(r)=-\frac{\varepsilon \zeta}{\mu} E_{z}\left(1-\frac{I_{\mathrm{o}}(\kappa r)}{I_{\mathrm{o}}(\kappa w)}\right) .
$$

The volume flow rate $Q$ can be obtained by integrating (2) over the cross-sectional area of the capillary or pore channel to give

$$
Q=\pi w^{2} \Omega E_{z}\left(1-\frac{I_{\mathrm{o}}(\kappa r)}{I_{\mathrm{o}}(\kappa w)}\right),
$$

where the ionic mobility is given by $\Omega=\varepsilon \varsigma / \mu$. Equation (2) can be simplify for capillaries with radius much larger than the double-layer thickness to

$$
Q=\pi w^{2} \Omega E_{z} .
$$

The pressure achieved in a plugged capillary can be obtained by first solving (1) as

$$
v_{z}(r)=\frac{P_{z}}{4 \mu}\left(w^{2}-r^{2}\right)-\frac{\varepsilon \zeta}{\mu} E_{z}\left(1-\frac{I_{\mathrm{o}}(\kappa r)}{I_{\mathrm{o}}(\kappa w)}\right),
$$

and then integrating to obtain the flow rate $Q$ similarly to (3). For such a plugged capillary, the net flow rate is zero. Using this condition, the solution can be obtained for the pressure gradient as

$$
P_{z}=8 \mu \Omega\left(1-\frac{I_{\mathrm{o}}(\kappa r)}{I_{\mathrm{o}}(\kappa w)}\right) \frac{E_{z}}{w^{2}} .
$$

Again, for $\kappa w>1,(6)$ can be simplified to

$$
P_{z}=8 \mu \Omega \frac{E_{z}}{w^{2}} .
$$

These scalings are for a single channel. For a channel of radius $R$ consisting of $(R / w)^{2}$ individual pores each of radius $w$, the flow rate in (4) becomes

$$
Q=\pi R^{2} \Omega E_{z}
$$

with the resulting flow speed $U=Q / \pi R^{2}$, and the force $F$ achieved in a plugged actuator of length $L$, from which the flow rate $Q$ is zero, becomes

$$
F=8 \pi \mu \Omega\left(\frac{R}{w}\right)^{2} E_{z} L .
$$

Note in (8) that the flow rate achieved is independent of the pore radius $w$, but the force in (9) increases as the pores are made smaller.

\subsection{Unsteady EK response modeling}

A simple hydrodynamical model has been proposed by Diez-Garias [20] and Diez and Dahm [25] to study the ultimate frequency response limits of unsteady electrokinetic microactuators. This limit applies when the electrokinetic double layer thickness is much smaller than the pore radius $w$, as is typically the case. The motion induced in the double layer by either an impulsively applied electric field, or by a sinusoidally oscillating applied field, is then equivalent, respectively, to Stokes' first or second problem for the flow induced by viscous diffusion above a moving wall. Stokes' second problem, which is most directly associated with the concept of frequency response, applies to the motion induced in the bulk electrolyte outside the double layer by a sinusoidally time-varying applied electric field, which produces a sinusoidal motion in the thin double layer directly adjacent to the wall. This motion is confined to a 'Stokes layer' with thickness $\delta \approx \sqrt{v t}$. The resulting velocity field in this case is self-similar, and given by

$$
u(y, t)=U_{\text {eq }} \mathrm{e}^{-\eta} \cos (\omega t-\eta),
$$

where $\eta \equiv y / \sqrt{2 v / \omega}$ is the wall-normal similarity coordinate. 


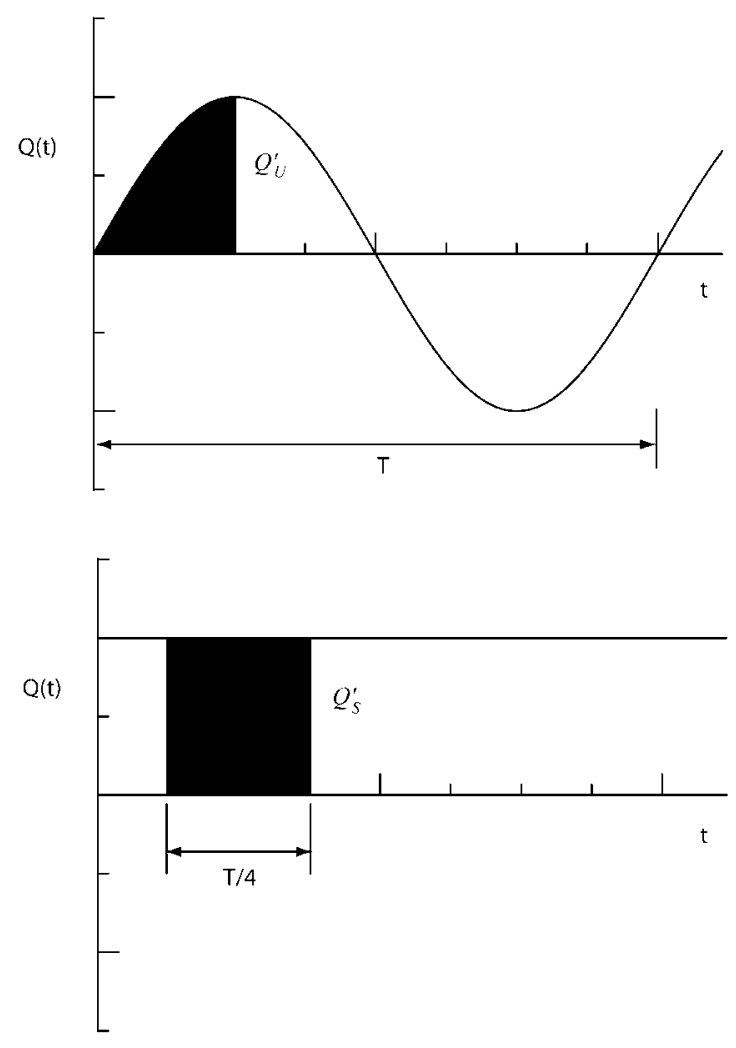

Figure 5. Schematics defining (a) the volume $Q_{\mathrm{U}}^{\prime}$ displaced by electrokinetic pumping per actuator stroke time $T / 4$ under a sinusoidally-applied electric field, and $(b)$ the volume $Q_{\mathrm{S}}^{\prime}$ displaced during the same time $T / 4$ under a steady applied electric field. Note that for time $T / 4$, the ratio of the two volumes is $Q_{\mathrm{U}}^{\prime} / Q_{\mathrm{S}}^{\prime} \approx 0.6$.

Figures 5(a) and (b) respectively define the actual flow rate $Q_{\mathrm{U}}^{\prime}$ that results from such a sinusoidally induced motion during each volumetric displacement 'stroke' of the actuator, and the flow rate $Q_{\mathrm{S}}^{\prime}$ that would result during the same time by steady electrokinetic pumping. Inertial effects in the induced flow are negligible when 'quasi-steady' performance is achieved, namely when $Q_{\mathrm{U}}^{\prime} / Q_{\mathrm{S}}^{\prime}=0.6$. The flow rate $Q_{\mathrm{U}}^{\prime}$ for a circular channel or pore is

$$
Q_{\mathrm{U}}^{\prime} \equiv \int_{t_{\mathrm{o}}}^{t_{\mathrm{o}}+T / 4} \int_{0}^{w} u(r, t) 2 \pi r(\mathrm{~d} r) \mathrm{d} t,
$$

which can be approximated by

$$
Q_{\mathrm{U}}^{\prime} \approx \frac{T}{4} 2 \pi w \delta U_{\mathrm{eq}}^{\mathrm{U}}
$$

where $U_{\text {eq }}^{\mathrm{U}}$ is the average velocity in the Stokes' layer directly adjacent to the wall, and $T$ is the period corresponding to each stroke of the actuator. An electrokinetic microactuator of radius $R$ filled with a porous matrix with pore size $w$ contains $N \approx(R / w)^{2}$ pores. From (12) the total flow rate $Q_{\mathrm{U}}^{\prime}$ produced by these $N$ pores in the entire actuator becomes

$$
Q_{\mathrm{U}}^{\prime} \approx \frac{\pi^{2}}{\sqrt{2}} \frac{R^{2}}{w} v^{1 / 2} \omega^{-3 / 2} U_{\mathrm{eq}}^{\mathrm{U}} .
$$

When a single capillary or pore is driven by a steady (S) field, as shown in figure $5(b)$, then the resulting flow rate $Q_{\mathrm{S}}^{\prime}$ is

$$
Q_{\mathrm{S}}^{\prime}=\frac{T}{4} \pi w^{2} U_{\mathrm{eq}}^{\mathrm{S}} .
$$

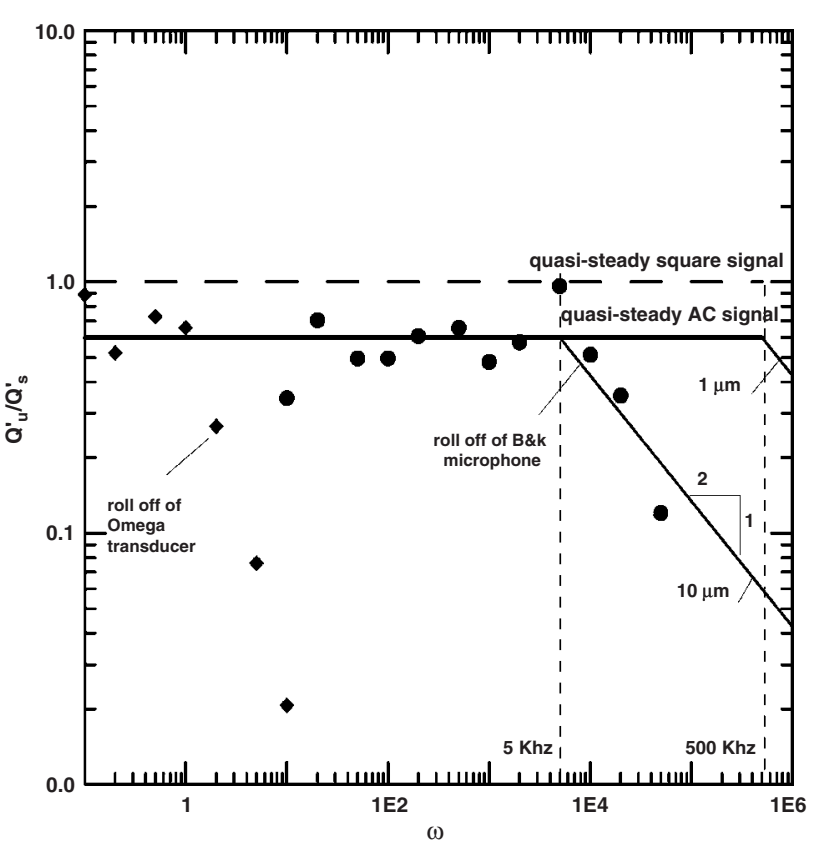

Figure 6. The ratio of $Q_{\mathrm{U}}^{\prime}$ to $Q_{\mathrm{S}}^{\prime}$, defined in figure 5, from (16) for any sinusoidal component of frequency $\omega$ in an unsteady applied electrif field. For $Q_{\mathrm{U}}^{\prime} / Q_{\mathrm{S}}^{\prime} \approx 0.6$, volumetric displacement achieved by electrokinetic pumping is in the quasi-steady limit with no losses due to inertia effects. Note that for $1 \mu \mathrm{m}$ pore sizes, roll-off produced by inertial effects does not begin until frequencies above $500 \mathrm{kHz}$.

Similarly, when the entire actuator is driven by a steady (S) field, as shown in figure $5(b)$ the total flow rate $Q_{\mathrm{S}}^{\prime}$ can be written as

$$
Q_{\mathrm{S}}^{\prime}=\frac{\pi^{2}}{2} R^{2} \omega^{-1} U_{\mathrm{eq}}^{\mathrm{S}} .
$$

The ratio of these two flow rates then allows the influence of inertial effects to be evaluated as

$$
\frac{Q_{\mathrm{U}}^{\prime}}{Q_{\mathrm{S}}^{\prime}} \approx \sqrt{2} \frac{v^{1 / 2}}{w} \omega^{-1 / 2} \frac{U_{\mathrm{eq}}^{\mathrm{U}}}{U_{\mathrm{eq}}^{\mathrm{S}}} .
$$

From (15) a cut-off frequency $\omega_{\mathrm{c}} \equiv v /\left(2 w^{2}\right)$ can be deduced below which the actuators will show 'quasi-steady' performance, and above which a roll-off will occur as shown in figure 6. Assuming an aqueous electrolyte with kinematic viscosity comparable to that of water $\left(v \approx 10^{-6} \mathrm{~m}^{2} \mathrm{~s}^{-1}\right)$, this indicates a cut-off frequency of the order of $500 \mathrm{kHz}$ for $1 \mu \mathrm{m}$ pores. For the porous polymer matrix used to fabricate the arrays in this study, the average pore size is roughly $1 \mu \mathrm{m}$, and this would suggest essentially loss-less frequency response up to around $500 \mathrm{kHz}$.

\subsection{Unsteady EK response measurement}

Unsteady performance testing was also conducted to determine frequency response, by applying a sinusoidally time-varying voltage difference on an electrokinetic pump (Diez-Garias [20] and Diez and Dahm [25]). Results indicate a loss-less unsteady performance up to $10 \mathrm{kHz}$ as shown in figure 6 . The origins of the roll-off above $10 \mathrm{kHz}$ remain unclear, and may be due to an experimental limitation. The measured frequency at which this roll-off begins would be consistent with the onset of 
inertial damping in a porous matrix with $10 \mu \mathrm{m}$ pores as shown in figure 6 , based on the previous considerations, whereas the nominal pore size in the electrokinetic driver channel is believed to be about $1 \mu \mathrm{m}$. These results demonstrate that the absolute (injected or removed) flow rate displaced by the actuators is given by the expression $Q_{\mathrm{U}}^{\prime} / Q_{\mathrm{S}}^{\prime}=0.6$ for frequencies up to $10 \mathrm{kHz}$ (current experiment) and $500 \mathrm{kHz}$ (for the simple theoretical model). Thus, these simple considerations suggest that it should be possible to meet the frequency response requirements and flowrate requirements, this last requirement was shown in figure 3 of Diez et al [25], for most of the vehicle types shown in table 1 and figure 2 by using microactuators with a porous matrix having typically $1 \mu \mathrm{m}$ pore radius in the electrokinetic channels.

\section{Electrokinetic microactuator array fabrication and testing}

\subsection{Elementary packed-capillary actuators}

Initial experiments with electrokinetic flow as the fundamental basis for a new class of microactuator arrays were performed using simple packed capillaries to provide the double-layer surface that drives the flow. Single electrokinetic micropumps have been characterized in great detail by Paul et al [30], and Gang et al [31], among others. Our initial 'actuators' consisted of glass capillaries with $100 \mu \mathrm{m}$ inner diameter, packed with glass beads of various diameters ranging from $0.5 \mu \mathrm{m}$ to $5 \mu \mathrm{m}$, and filled with various aqueous electrolytes that provided differing zeta potentials. The glass beads created effective interstitial channels at submicron scales. The purpose of these experiments was to verify the applicability of the scaling results in section 4 for such packed-capillary electrokinetic pumps at these scales under steady (S) applied fields.

Results verified the $Q \sim w^{2} E_{z}$ scaling of the flow rate $Q$ for single microchannels with diameter $w$ and applied field strength $E_{\mathrm{z}}$, and the $\Delta p \sim E_{z} L / w^{2}$ scaling, to pore sizes $w$ at least as small as those produced by the $0.5 \mu \mathrm{m}$ glass beads [25]. In view of the exceedingly small Debye length scales associated with the double-layer thickness, which typically is in the range of $10-100 \mathrm{~nm}$, the observed applicability of these scalings even at the smallest bead diameter $(500 \mathrm{~nm})$ is not entirely surprising. Nevertheless, the results confirmed that fabrication of electrokinetic microactuators with pore sizes down to at least these scales does not invalidate these fundamental performance scaling laws. This indicates that electrokinetic microactuators based on porous matrix structures having average pore sizes of $1 \mu \mathrm{m}$, for which the entire range of pore sizes should be within the range applicable to the tests above using $0.5 \mu \mathrm{m}$ beads, should allow large values of pressure rise $\Delta p$ to be achieved in plugged actuators, while causing essentially no reduction in volume displacement rate $Q$ for open actuators. All subsequent micro-electro-kineticactuator (MEKA) arrays developed in this study were based on electrokinetic flow produced in such micron-scale porous matrix structures.

\subsection{MEKA-0 microactuator array}

Based on the results from experiments with elementary packed channel actuators, an initial $3 \times 3$ array of electrokinetic

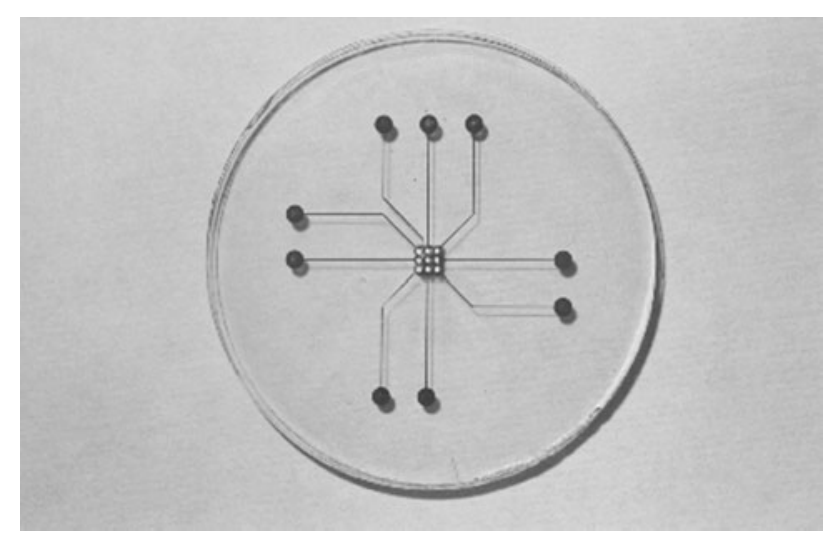

(a)

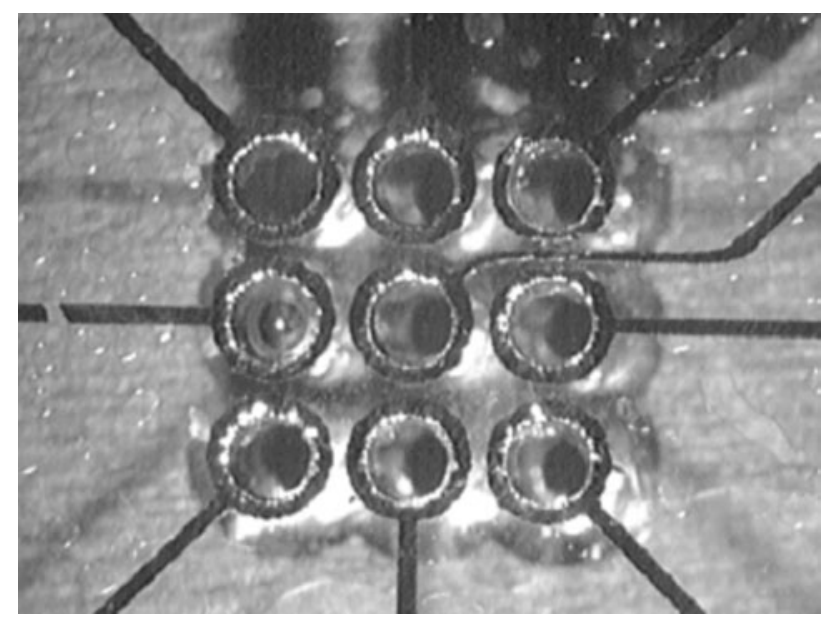

(b)

Figure 7. MEKA-0 electrokinetic microactuator array, showing (a) array fabricated in glass, and (b) closeup view prior to polymer filling, showing top-surface ring electrodes for each actuator.

microactuators, shown in figures 7(a) and (b), was fabricated and used for performance testing. This array is somewhat smaller than the typical $4 \times 4$ unit cell indicated in figure 3 , however its purpose was principally for an initial exploration of the major challenges involved in unit-cell fabrication, and for initial steady (S) performance testing of electrokinetic flows. For added simplicity, this initial array was sized to the requirements of the UAV-scale application in figure 2, and thus consisted of individual microactuators with $1000 \mu \mathrm{m}$ diameters and $2000 \mu \mathrm{m}$ center-to-center spacings. A basic three-layer design shown in figure 3 was used, with electrokinetic driver channels in the center layer and an electrolyte reservoir in the bottom layer, but with electrodes and leadouts formed on the top surface of the center layer. This basic three-layer design allows for dense arrays comprised large numbers of such actuators to be fabricated in thin conformally applicable tiles that accommodate the unit-cell system architecture, and that may in principle be easily applied to the surface of real vehicles. Elements of this basic threelayer design have been developed in successive generations of electrokinetic microactuator arrays described in the following sections.

The electrokinetic driver channels for the MEKA- 0 array were in this case mechanically drilled into a $3 \mathrm{~mm}$ thick glass 
substrate. This was intended as a preliminary process used for this initial fabrication only, and not intended to represent a fabrication process suitable for eventual mass production. An individual ring electrode with a lead out to an electrical contact was formed on the top surface around the periphery of each of the microactuator channels using standard metallization and photoetching techniques, as can be seen in figure 7(a). Due to chipping that occurred in the channel drilling process, as shown in figure $7(b)$, the resulting edge quality was relatively poor. This caused some difficulties in maintaining electrode continuity on the top surface of the center layer, however the resulting ring electrodes were still of acceptable quality. The bottom surface electrode is common for all the channels.

The individual center-layer electrokinetic driver channels shown in figure $7(b)$ were filled with the porous polymer matrix structure developed by Peters et al $[32,33]$. In the MEKA-0 array this polymer consisted of several constituents, including butyl methacrylate with a solvent and with a dopant to provide a zeta potential of $80 \mathrm{mV}$ in a $2 \mathrm{mM}$ sodium borate buffer solution. The resulting porous polymer matrix structure is visible as the white material in the channels in figure $7(a)$. This material has $1 \mu \mathrm{m}$ scale pore sizes and was doped to provide electrokinetic flow. The porous polymer can readily be wetted with electrolyte by simple capillary wicking action. Continual contact with the electrolyte, and thereby continual wetting of the entire porous matrix throughout each individual actuator channel in the center layer, is provided by a common electrolyte reservoir in the bottom layer. Since each unit cell acts as a zero-net-displacement entity, a fixed amount of electrolyte is maintained within each unit cell.

Steady performance testing was conducted using the apparatus shown in figure $8(a)$. This consisted of an electrolyte reservoir, $85 \mathrm{~mm}$ in diameter by $2.5 \mathrm{~mm}$ in height, which had the MEKA-1 array wafer located above it. The reservoir was filled with a $2 \mathrm{mM}$ sodium borate buffer solution. When a steady voltage difference $\Delta V$ was applied across the electrodes, the electrokinetic actuators steadily pumped the electrolyte into the graduated reservoir shown in figure $8(a)$. The volume flow rate was measured over an extended time and the results are shown in figure $8(b)$. Note that the total volume displaced increased linearly with time, with the flow rate $Q_{\mathrm{S}}$ being given by the slope of each set of symbols. The flow rate achieved is seen to be proportional to the applied voltage, consistent with (1). For these conditions, the porous polymer matrix material provided a volume flow rate $Q_{\mathrm{S}}$ of $0.1 \mathrm{~mL} \mathrm{~min}^{-1}$ at an applied field strength $E_{\mathrm{S}}$ of $15.3 \mathrm{~V} \mathrm{~mm}^{-1}$ for an electrokinetic actuator with an effective radius of $1.5 \mathrm{~mm}$. The ratio $\left(Q_{\mathrm{S}} / E_{\mathrm{S}}\right)$ should thus be independent of the field strength, and represents the fundamental characterization of the electrokinetic pumping capability of the porous polymer matrix structure per unit cross-sectional area of the actuator channels.

\subsection{MEKA-1 microactuator array}

Based on the performance seen with the MEKA-0 microactuator array in section 5.2, a MEKA-1 array was fabricated to (i) assess laser drilling of the electrokinetic channels as an alternative to mechanical drilling, and (ii) explore design issues associated with larger unit cells. This

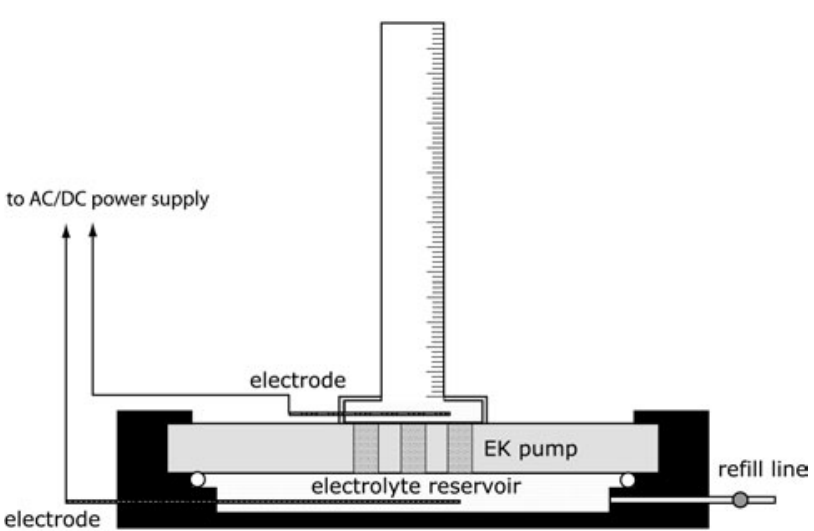

(a)

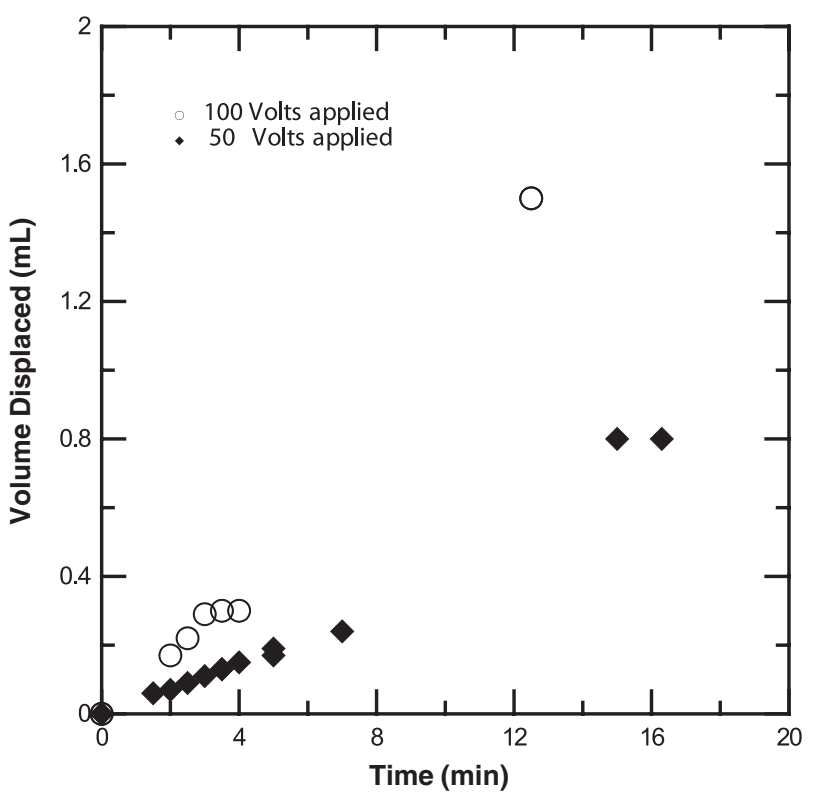

(b)

Figure 8. (a) Schematic of experimental apparatus used for steady flow rate measurement with MEKA-0 array, and $(b)$ experimental results for steady electrokinetic pumping performance achieved with MEKA-0 array for two different applied voltages.

$10 \times 10$ actuator array was fabricated in glass, as was the case for the previous MEKA-0 array, and again sized for a UAVscale application. The individual electrokinetic microactuator channels were $2000 \mu \mathrm{m}$ in diameter and located on $4 \mathrm{~mm}$ center-to-center spacings. The layout for this MEKA-1 array is shown in figure $9(a)$. Drilling of the electrokinetic driver channels in glass was in this case done with a $\mathrm{CO}_{2}$ laser by a commercial vendor. This fabrication process is amenable to eventual mass production. Edge quality achieved at this scale with laser drilling was excellent, and more than adequate for metallization and electrode etching. The $10 \times 10$ unitcell size of this MEKA-1 array is considerably larger than the $4 \times 4$ unit-cell anticipated in figure 3 for the proposed system architecture, yet figure $9(a)$ shows that the required electrode leadouts can readily be accommodated. Like the MEKA-0 array, the bottom surface electrode is common for all channels. The channels were filled with the same porous polymer matrix as for the MEKA-0 array. Figure $9(b)$ shows 


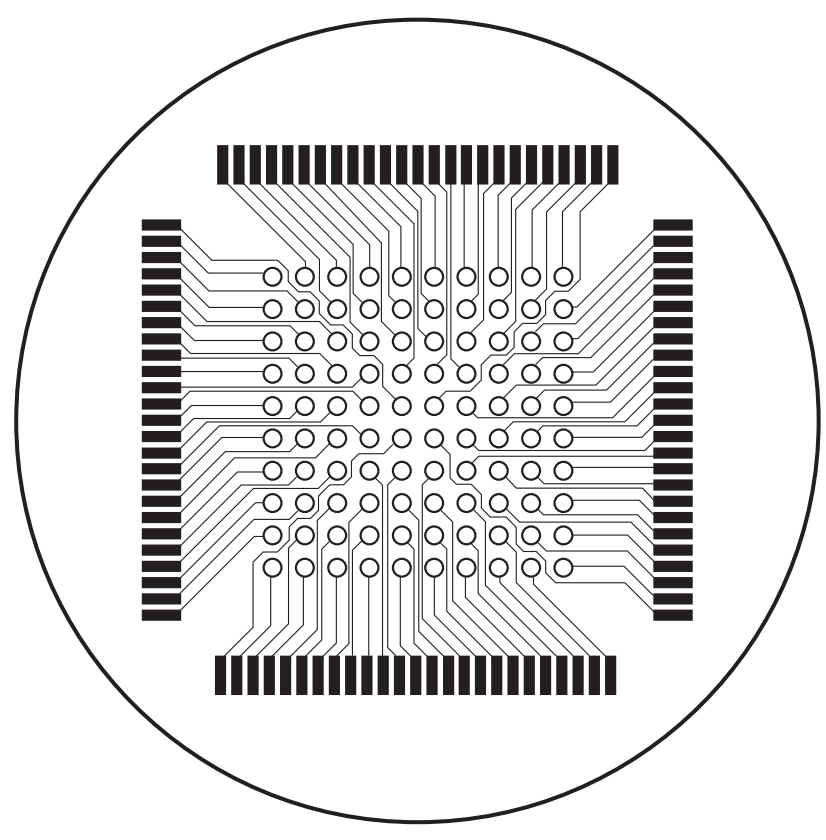

(a)

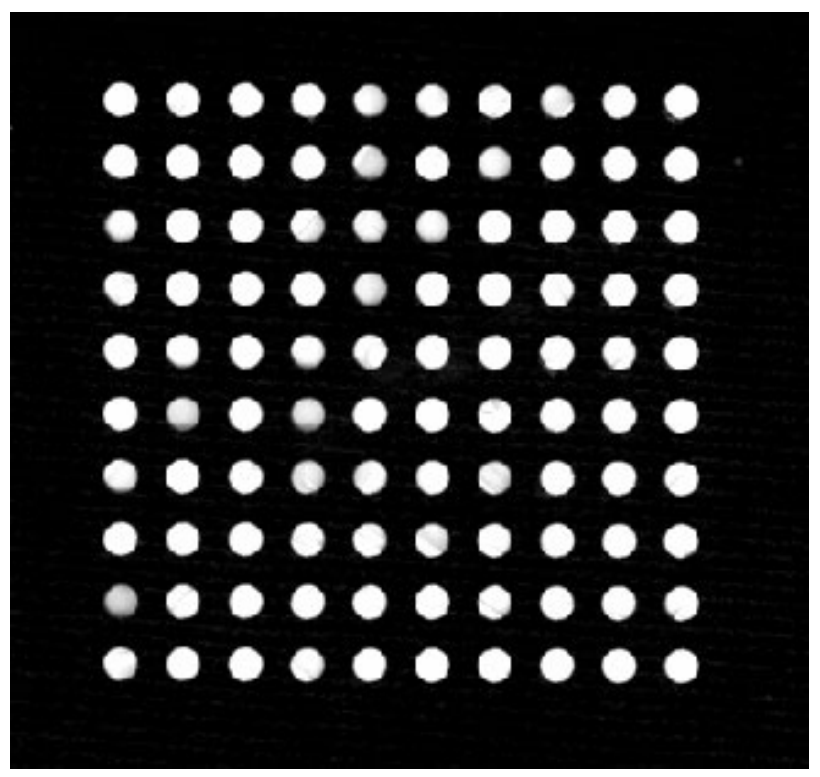

(b)

Figure 9. (a) Electrode and leadout pattern for MEKA-1 array showing individual top-surface ring electrode and leadout for each individual actuator channel, and $(b)$ photograph of $1 \mu \mathrm{m}$ porous polymer matrix structure filled in MEKA-1 array.

the resulting filled microactuator channels, where the $1 \mu \mathrm{m}$ scale porous polymer matrix structure again appears white.

\subsection{MEKA-2 microactuator array}

In view of the excellent edge quality achieved by the laser drilling process in the UAV-scale MEKA-1 array, the process was applied to develop a hydronautical-scale MEKA-2 array. The principal purpose of this array was to test the suitability of the $\mathrm{CO}_{2}$ laser drilling process in a glass substrate at much smaller scales. Accordingly, this array consisted of a simple
$3 \times 3$ matrix of electrokinetic driver channels, each with $300 \mu \mathrm{m}$ diameter and $2 \mathrm{~mm}$ length, on $500 \mu \mathrm{m}$ center-tocenter spacings. The smaller channel diameters in this case produced much poorer results from laser drilling than was the case for the $2000 \mu \mathrm{m}$ diameter channels in the MEKA- 1 array. These difficulties were driven by fabrication glass substrate material. Since glass was not intended as a final substrate material, subsequent arrays examined fabrication with other substrate materials.

\subsection{MEKA-3 microactuator array}

The $\mathrm{CO}_{2}$ laser drilling process was adapted for a MEKA-3 array by changing the substrate material from glass to acrylic, which is also readily amenable to laser drilling. The MEKA-3 array, consisted of a $5 \times 5$ matrix of electrokinetic driver channels, each $300 \mu \mathrm{m}$ in diameter and $2 \mathrm{~mm}$ long, and with $1000 \mu \mathrm{m}$ center-to-center spacings. The results obtained from this MEKA-3 fabrication with laser drilling in acrylic indicate that it is possible to mass produce the center layer in thin plastic films by such a process. However, acrylic is unsuitable as a center-layer substrate material since it is incompatible with the organic solvents used in the porous polymer process. There are other plastics that can readily be laser drilled and that are compatible with these solvents. For this reason, the center layer for MEKA-5 array in the following section was produced in mylar, which can be laser drilled and is suitable for use with the porous polymer process.

\subsection{MEKA-5 hydronautical array}

5.6.1. MEKA-5 array design. The MEKA-5 actuator array, shown in figure 10, demonstrated fabrication of a full hydronautical-scale array of electrokinetic microactuators and their integration with a top layer containing the basic unitcell structure and all electrical leadouts required for actuation. The array was fabricated in a $7 \times 7 \mathrm{~cm}^{2}$ tile, containing 25600 individual electrokinetic microactuators with $250 \mu \mathrm{m}$ channel diameters arranged on $350 \mu \mathrm{m}$ center-to-center spacings, as indicated in figure 2 for full-scale hydronautical vehicle applications. These individual electrokinetic microactuators were grouped into 1600 individual unit cells arranged in a $40 \times 40$ unit-cell pattern on each tile, with each unit-cell composed of a $4 \times 4$ array of microactuators. As indicated in figure 10 , every fifth row and column of microactuators in the tile was skipped to provide room for the resident unit-cell processing electronics. The top layer, shown in figure 11(a), was fabricated using conventional MEMS processing steps. The center layer in figure $11(b)$ was fabricated with the same porous polymer matrix material in thin flexible mylar substrate material suitable for conformal application on a vehicle surface. The following sections describe these centerlayer and top-layer fabrication processes used to develop this full-scale MEKA-5 hydronautical array.

5.6.2. MEKA-5 center-layerfabrication. Figure 12(a) shows the center layer of the three-layer MEKA-5 hydronautical array tile. Each of the 25600 individual electrokinetic microactuators has a $250 \mu \mathrm{m}$ microchannel diameter and $350 \mu \mathrm{m}$ center-to-center spacing between microactuators 


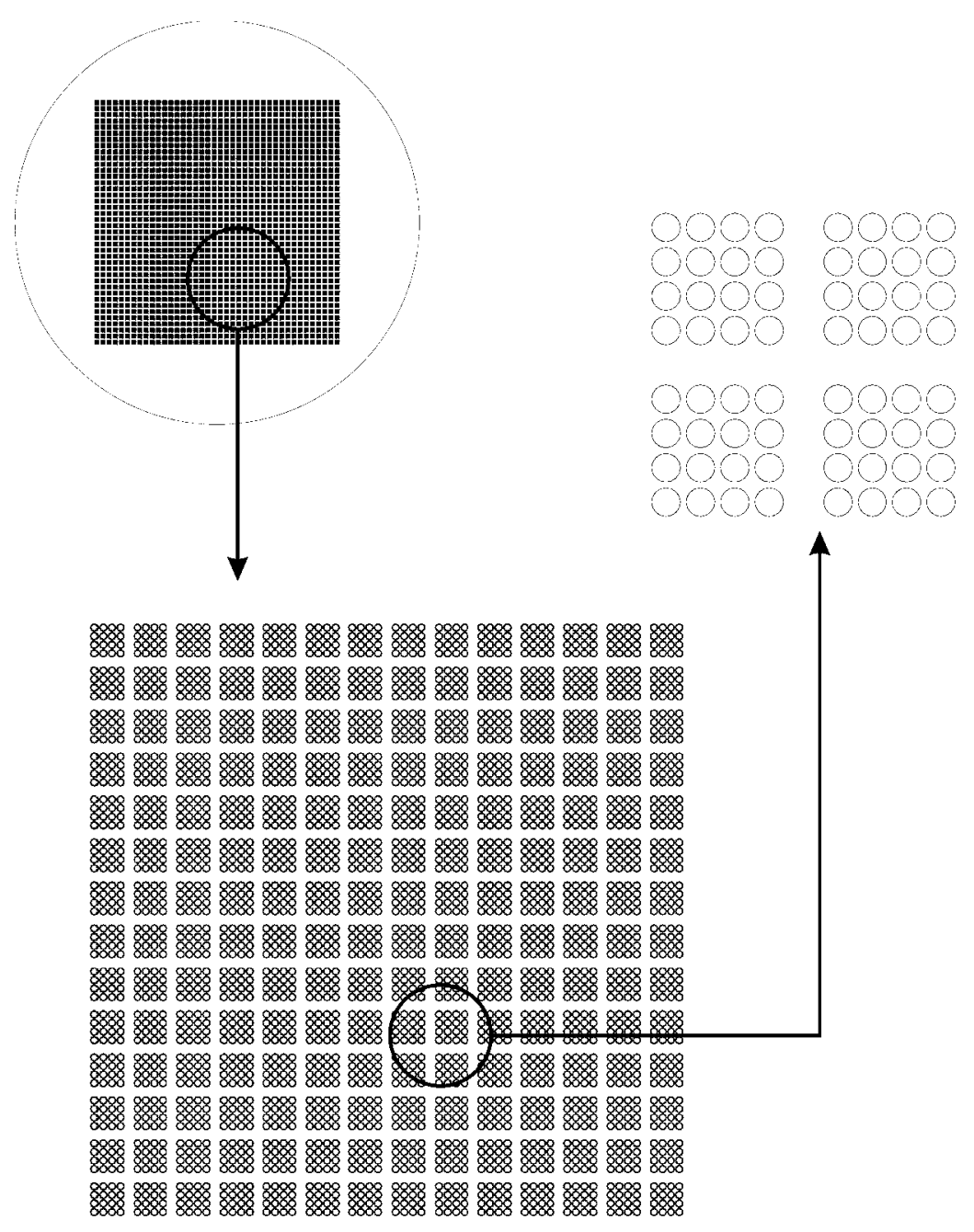

Figure 10. Unit-cell pattern on the MEKA-5 hydronautical-scale array, showing how full tile is arranged into individual $4 \times 4$ unit-cell structures. Every fifth row and column of actuators was skipped to provide space for unit-cell control processing.

Top Layer
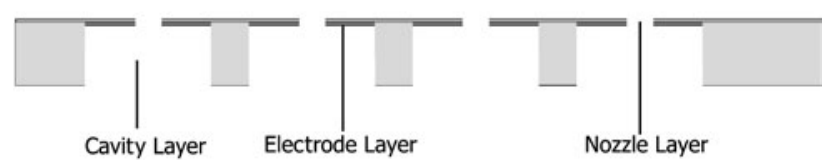

Middle Layer

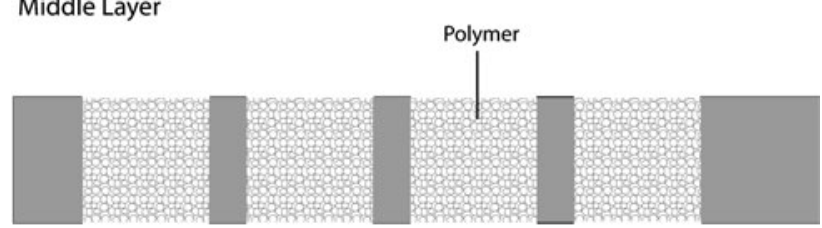

Figure 11. Basic elements fabricated in 25 600-element MEKA-5 hydronautical-scale array, showing $(a)$ top layer with cavities, electrodes and leadouts, and nozzles, and $(b)$ center layer with actuator channel and porous polymer matrix.

within a unit cell. The channels were fabricated in flexible mylar substrate material, having $250 \mu$ m thickness to permit a high electric field $E$ across the individual actuator channels at a low voltage difference. A potential difference of just $20 \mathrm{~V}$ across this $250 \mu \mathrm{m}$ thick center layer produces the necessary field strength to achieve the $10 \mu \mathrm{L} \mathrm{min}^{-1}$ equivalent steady (S) flow rate needed for active sublayer control on hydronautical vehicles, given the pumping capability of the porous polymer demonstrated in figure $8(b)$. The electrokinetic porous polymer matrix structure was filled in the liquid state in the microchannels by a two-component polymerization process $[32,33]$. The curing process produced a porous matrix with pore sizes in the range of $1 \mu \mathrm{m}$. Figure 12(b) shows an SEM image of the typical resulting pore structure. Note that pore sizes vary significantly, but are typically $1 \mu \mathrm{m}$ or smaller, indicating a roughly $1 \mathrm{MHz}$ theoretical frequency response limit based on the considerations in section 4 . The $10 \mathrm{kHz}$ loss-less frequency response demonstrated with essentially the same porous polymer matrix by Diez-Garias [20] and Diez and Dahm [25] is thus more than sufficient for the $1 \mathrm{kHz}$ frequency response requirements in figure 2 for sublayer control on large hydronautical vehicles.

5.6.3. MEKA-5 top layer. The top layer of the MEKA-5 array was fabricated using photolithographic etching and other MEMS mass fabrication processes. This layer provides the 25600 individual microactuator electrodes and leadouts, grouped into a unit-cell architecture as shown in figure 13. 


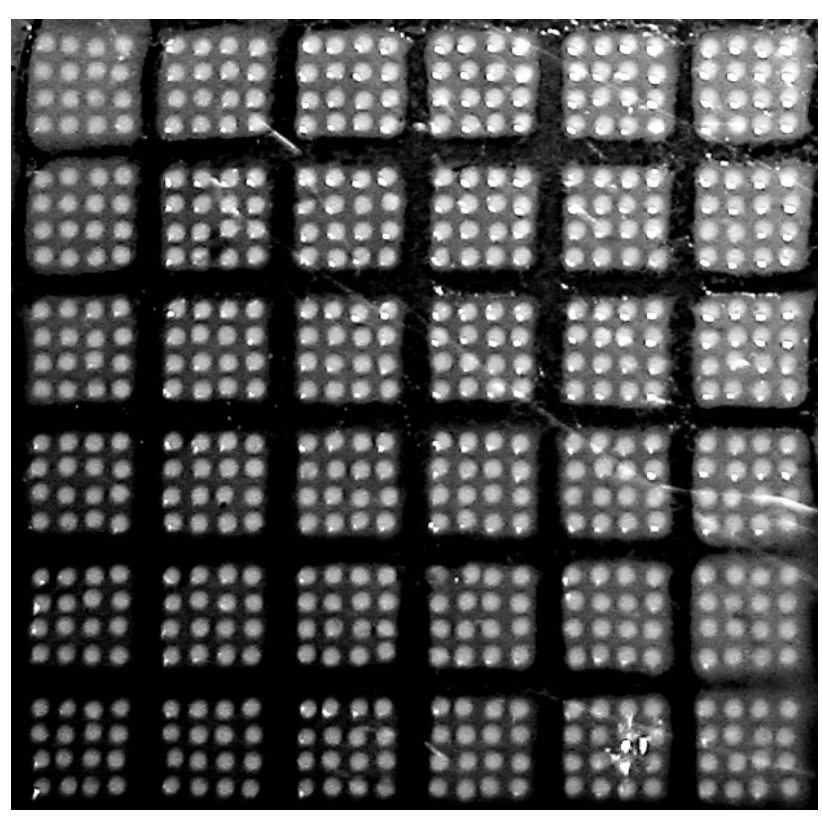

(a)

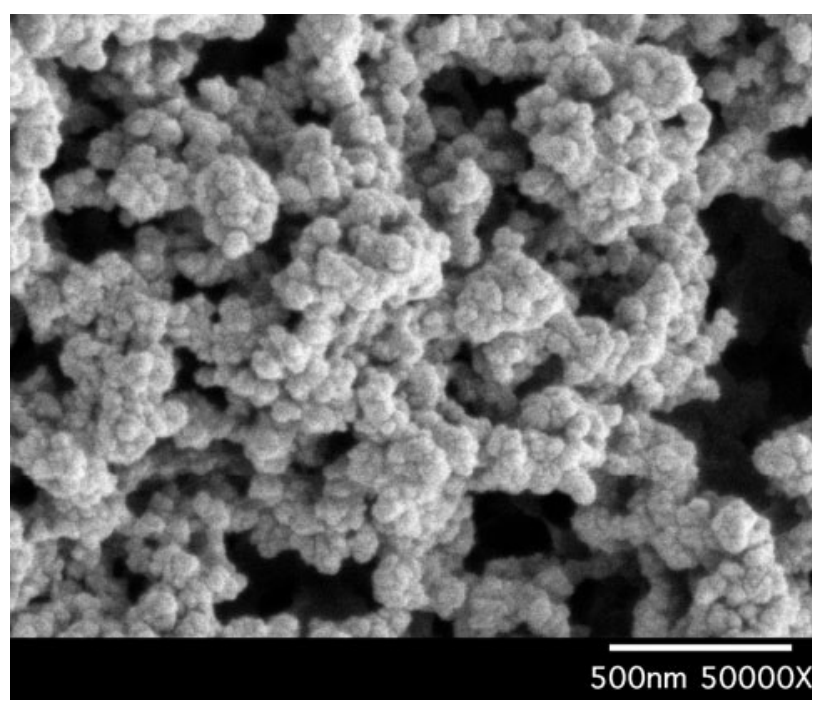

(b)

Figure 12. (a) Center layer of 25600 -element MEKA-5 hydronautical-scale array fabricated in thin flexible mylar, showing individual $4 \times 4$ unit-cells and closeup view of $250 \mu \mathrm{m}$ diameter actuator channels filled with porous polymer matrix structure. (b) SEM micron-scale photograph of the porous polymer matrix structure resulting from two-component polymer fabrication process of the type used in the MEKA-5 array.

The top layer was fabricated by a three-mask MEMS process consisting of an 'electrodes mask', a 'cavities mask', and a 'nozzles mask'. The fabrication process sequence developed for this MEMS top layer is shown in figure 14. A $525 \mu \mathrm{m}$ silicon wafer was first used as a temporary substrate for the top layer. A $2 \mu \mathrm{m}$ thick layer of aluminum was then sputtered on the top surface of this wafer-the entire MEKA-5 top layer was fabricated on top of this aluminum layer. The silicon wafer was used solely to provide support for the top layer and to permit handling during the fabrication process. Upon completion of the fabrication process described below, this

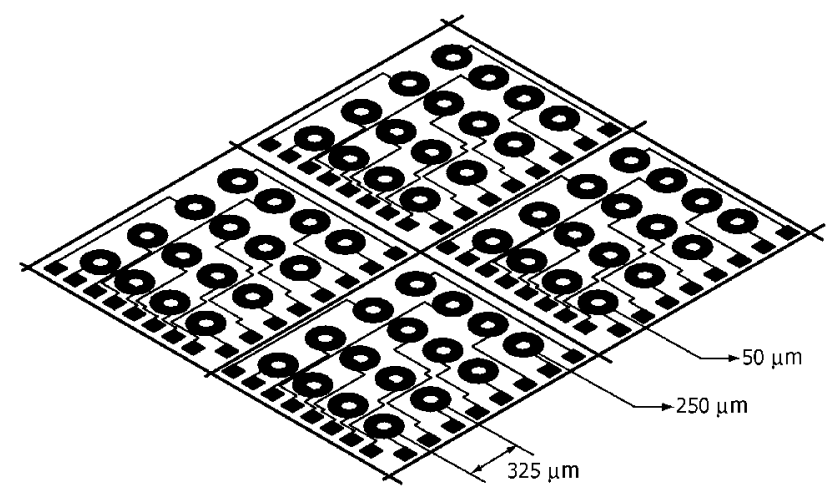

Figure 13. Layout of top-surface electrodes sublayer mask for 25 600-element MEKA-5 array, showing disk electrodes with leadouts to contacts near edge of each unit cell, and common voltage bus lines running horizontally and vertically between adjacent unit cells.

aluminum layer was etched away as shown in the last step in figure $14(j)$, to release the top layer from the silicon wafer.

As regards the fabrication of the top layer itself, a $6 \mu \mathrm{m}$ thick layer of polyimide was first spin coated over the aluminum layer. Using the nozzles mask, the polyimide layer was then photolithographically patterned, unmasked and exposed to define the $50 \mu \mathrm{m}$ nozzle array. A layer of photoresist was then used to coat the entire surface. Using the electrode mask, corresponding parts of this photoresist layer were then etched away. This defined the areas where the electrodes were to be located. A $4000 \AA$ layer of chrome was then sputtered onto this surface, followed by another $4000 \AA$ layer of gold. The use of $\mathrm{Cr} / \mathrm{Au}$ for the electrodes proved to be important for the fabrication process, as early attempts to pattern the electrodes using platinum repeatedly failed because this material would consistently peel away from the underlying polyimide layer. A similar result occurred when gold alone was used. This $\mathrm{Cr} / \mathrm{Au}$ double layer was then patterned and unmasked using the electrode mask, and the photoresist then etched with acetone to expose the electrodes. Finally, a $125 \mu \mathrm{m}$ thick layer of Su-8 material was deposited and selectively etched using the cavities mask. At this point, the top-layer fabrication was complete, and the layer was released from the silicon support wafer by etching away the aluminum layer with a phosphoric-nitric-acetic acid mixture.

The three-component sublayers that make up the top layer are named after the masks used to fabricate them. A full-tile view of the 'electrodes sublayer' is shown in figure 15(a). This sublayer provides $\mathrm{Cr} / \mathrm{Au}$ electrodes for each of the 25600 individual microactuators and the corresponding leadout for each actuator to a contact located near the edge of its unit cell. A region of this electrodes sublayer is shown in figure $15(b)$ where the repeated pattern in 20 adjacent units cells can be seen. A closer view of a single unit cell in this structure is shown in figure $15(c)$ with the 16 unit-cell contacts for each of the individual electrodes in the unit cell, together with the common voltage bus lines that run between adjacent unit cells. Future versions of this MEKA-5 array could contain a digital signal processing layer that selectively connects each of these contacts to the bus line with either positive or negative polarity, depending on the actuation state vector implied by the sensor states. 


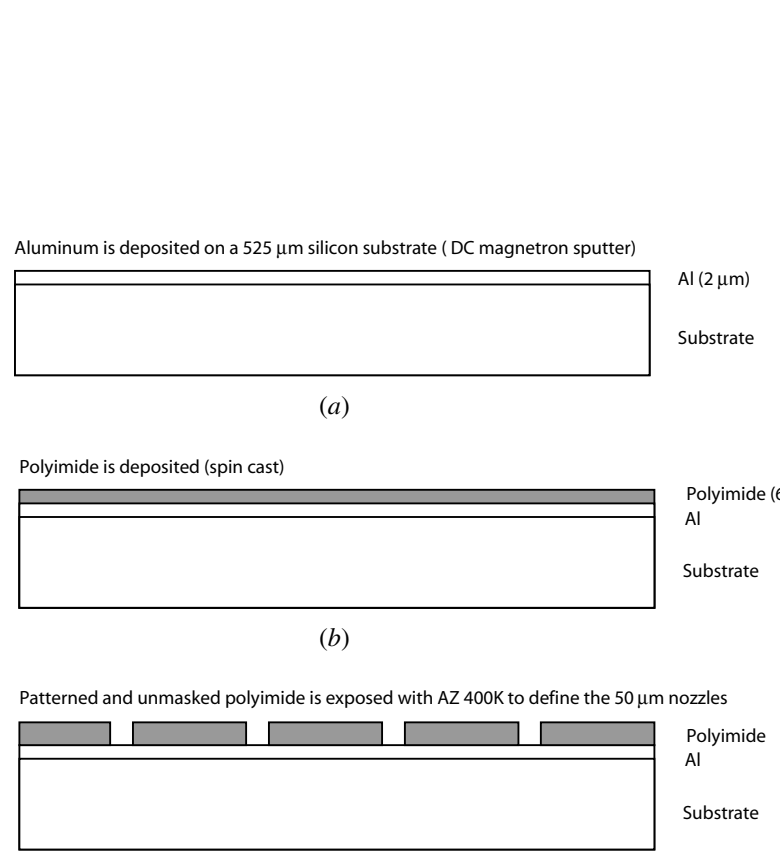

(c)

Photoresist (Shipley 1827) coat is deposited (spin cast)

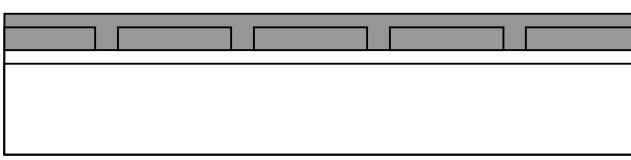

(d)

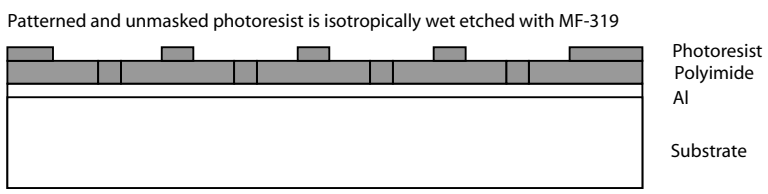

(e)

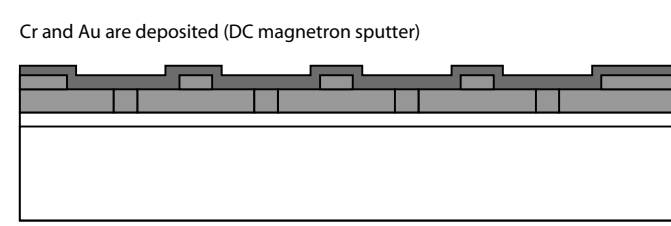

$\mathrm{Cr} / \mathrm{Au}(0.8 \mu \mathrm{m})$ Photoresist Polyimide Substrate

$(f)$

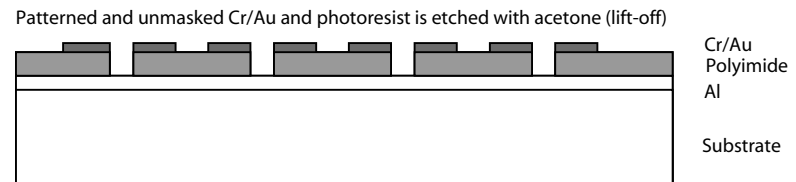

$(g)$

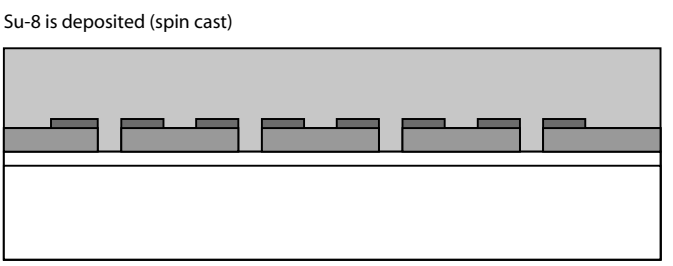

Su-8

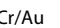
Polyimide Substrate

$(h)$

Patterned and unmasked Su-8 is etched with Su-8 developer

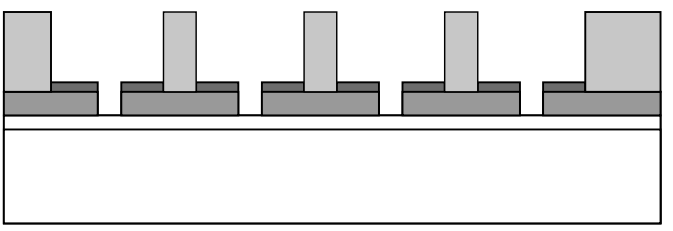

Su-8 $\mathrm{Cr} / \mathrm{Au}$ $\mathrm{Cr} / \mathrm{Au}$
Polyimide Al Substrate

(i)

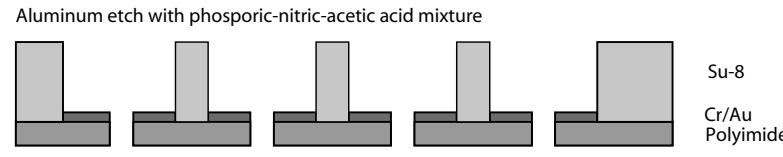

(j)

Figure 14. MEMS process sequence $(a)-(j)$ developed for top layer of MEKA-5 array.

The 'nozzles sublayer' is etched in polyamide and provides the $50 \mu \mathrm{m}$ nozzles through which the pumping induced by the electrokinetic flow occurs. The 'cavities sublayer' fabricated in clear Su-8 material as described in figure 14, serves two specific functions. It provides the necessary structural support for the top layer, and at the same time provides the necessary separation between the top of the porous polymer matrix structure associated with each microactuator channel in the center layer and the corresponding top-layer electrode. The cavities sublayer is composed of $250 \mu \mathrm{m}$ diameter cavities on $325 \mu \mathrm{m}$ center-tocenter spacings. The fabrication process shown in figure 14 produced comparatively well-defined edges and vertical walls for the circular cavity enclosures.

\section{Conclusion}

The present paper has (i) set forth the basic principles underlying the control of streamwise vortical structures in the viscous sublayer of turbulent boundary layers for drag reduction on vehicles, and (ii) demonstrated that the electrokinetic principle can provide the basis for a class of microactuator arrays to control the sublayer vortices. 'Point' microactuator arrays (of the type illustrated in figure 3) serve as volume sources to impulsively displace the sublayer vortices (as indicated schematically in figure 1) necessary to interrupt the interaction between adjacent structures and thereby delay or defeat the bursting process. Performance requirements for such microactuator arrays at various locations on different types of aeronautical and hydronautical vehicles were given in figure 2. A basic architecture comprised small, independent unit cells with local sensing and signal processing is appropriate for closed-loop control with point microactuator arrays. Fundamental considerations related to the physics of the electrokinetic process indicate the basic scaling laws that govern such microactuators. These show advantages obtained from miniaturization of the electrokinetic channels by use of a porous matrix with $1 \mu \mathrm{m}$ scale pores in the center layer of the proposed three-layer design. Simple hydrodynamic models for the electrokinetically driven flow in such a porous matrix suggest that inertial damping should remain irrelevant up to frequencies approaching $1 \mathrm{MHz}$.

Several microactuator arrays or components have been assembled to study various aspects of the performance and 


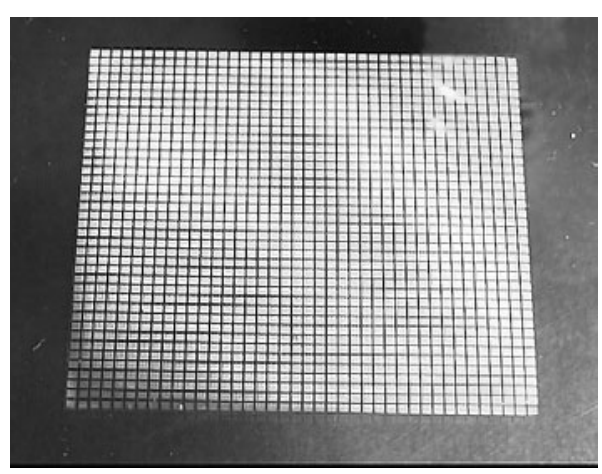

(a)

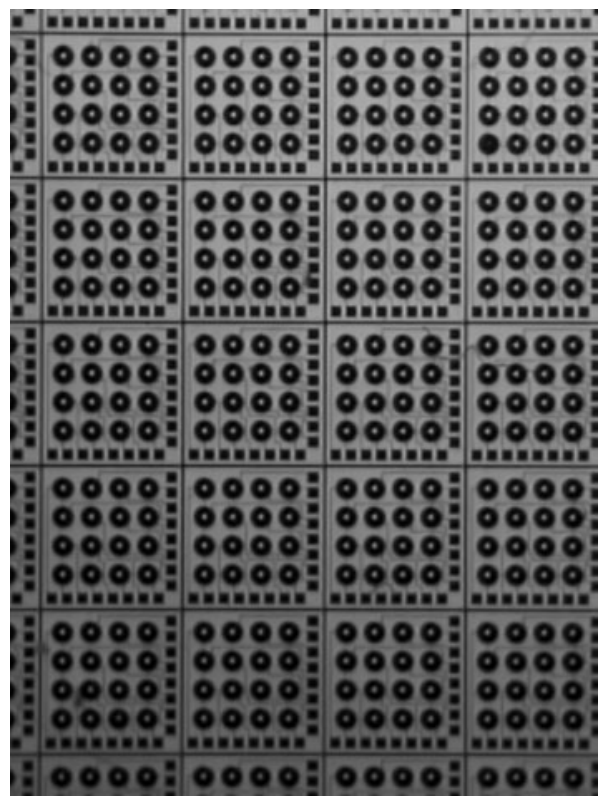

(b)

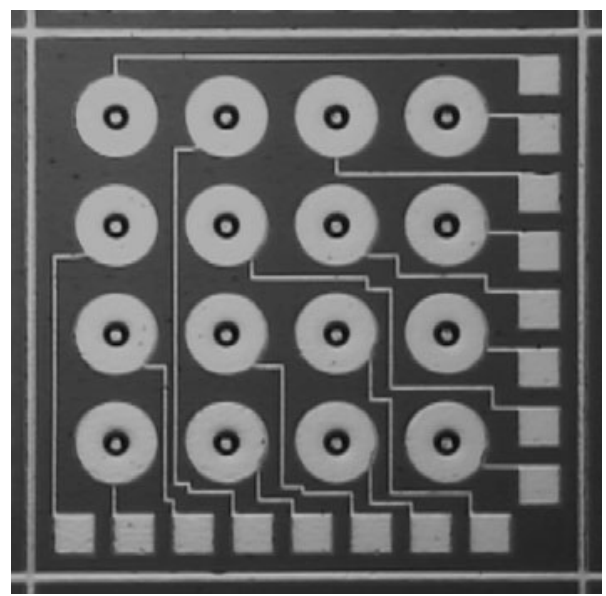

(c)

Figure 15. (a) Tile-scale view of electrodes sublayer fabcricated via the process sequence in figure 11 for the 25 600-element MEKA-5 hydronautical-scale array. (b) Closeup view of the resulting unit-cell structure in the electrodes sublayer. $(c)$ Unit-cell-scale view of the electrodes sublayer showing a single unit cell.

fabrication processes relevant to electrokinetic microactuator arrays. The MEKA- 0 array in figures $7(a)$ and $(b)$ consisted of an individually addressable $3 \times 3$ array that demonstrated good steady (S) performance. The porous polymer matrix permitted relatively easy fabrication of $\mu \mathrm{m}$-scale electrokinetic channels. These different fabrication steps have led to the MEKA-5 full-scale hydronautical array, composed of 25600 individual electrokinetic microactuators arranged in a $40 \times 40$ pattern of unit cells, each composed of a $4 \times 4$ matrix of actuators. Such arrays have been successfully fabricated in a $7 \times 7 \mathrm{~cm}^{2}$ tile in $250 \mu \mathrm{m}$ thick mylar substrate material. MEMS design and fabrication processes were used to produce a top layer for the MEKA-5 hydronautical-scale array, composed of an electrode sublayer patterned in $\mathrm{Cr} / \mathrm{Au}$, a nozzle sublayer etched in polyimide, and a cavities sublayer fabricated in Su-8; the functionality incorporated in this top-layer fabrication demonstrates the complete set of elements needed for microscale electrokinetic actuator arrays for sublayer control.

\section{Acknowledgments}

This work was supported by the Defense Advanced Research Projects Agency (DARPA) MEMS/MTO Program under Contract No. F30602-98-2-0228. The assistance of Dr Phillip $\mathrm{H}$ Paul and $\mathrm{Mr}$ Ken $\mathrm{R}$ Hencken of the Microtechnology Department at Sandia National Laboratories in fabrication and testing of the electrokinetic microactuators is gratefully acknowledged, as is the assistance of Professor Khalil Najafi and Mr Paul Sunal at The University of Michigan MEMS Exchange in fabricating the MEKA-5 array.

\section{References}

[1] Moin P and Bewley T 1994 Feedback control of turbulence Appl. Mech. Rev. 45 S3-S13

[2] Gad-el-Hak M 1996 Modern developments in flow control Appl. Mech. Rev. 49 365-79

[3] Gad-el-Hak M 2001 Flow control: the future J. Aircraft 38 402-18

[4] McMichael J M 1996 Progress and prospects for active flow control using microfabricated electromechanical systems (MEMS) AIAA Paper 96-0306 (Washington, DC: AIAA)

[5] Ho C-M and Tai Y-C 1998 Micro-electro-mechanical-systems (MEMS) and fluid flows Annu. Rev. Fluid Mech. 30 579-612

[6] Pollard A 1997 Passive and active control of near-wall turbulence Prog. Aerospace Sci. 33 689-708

[7] Lumley J L and Blossey P 1998 Control of turbulence Annu. Rev. Fluid Mech. 30 311-27

[8] Löfdahl L and Gad-el-Hak M 1999 MEMS applications in turbulence and flow control Prog. Aerospace Sci. 35 101-203

[9] Bewley T R 2001 Flow control: new challenges for a new Renaissance Prog. Aerospace Sci. 37 21-58

[10] Tsao T, Liu C, Tai Y-C and Ho C-M 1994 Micromachined magnetic actuator for active fluid control ASME Int. Mech. Eng. Congress and Expo. (Chicago, IL) pp 31-8

[11] Jacobson S A and Reynolds W C 1993 Active boundary layer control using flush mounted surface actuators Bull. Am. Phys. Soc. 382197

[12] Jacobson S A and Reynolds W C 1998 Active control of streamwise vortices and streaks in boundary layers J. Fluid Mech. 360 179-211

[13] Rathnasingham R and Breuer K S 1997 System identification and control of a turbulent boundary layer Phys. Fluids 9 1867-9 
[14] Myose R Y and Blackwelder R F 1995 Control of streamwise vortices using selective suction AIAA J. 33 1076-80

[15] Choi H, Temam R, Moin P and Kim J 1993 Feedback control for unsteady flow and its application to the stochastic Burgers equation J. Fluid Mech. 253 509-43

[16] Choi H, Moin P and Kim J 1994 Active turbulence control for drag reduction in wall-bounded flows J. Fluid Mech. $\mathbf{2 6 2}$ $75-110$

[17] Hammond E P, Bewley T R and Moin P 1998 Observed mechanisms for turbulence attenuation and enhancement in opposition-controlled wall-bounded flows Phys. Fluid $\mathbf{1 0}$ 2421-3

[18] Rebbeck H and Choi K-S 2001 Opposition control of near-wall turbulence with a piston-type actuator Phys. Fluids 13 2142-5

[19] Diez-Garias F J, Dahm W J A and Paul P H 2000 Microactuator arrays for sublayer control in turbulent boundary layers using the electrokinetic principle AIAA Paper 2000-0548 (Washington, DC: AIAA)

[20] Diez-Garias F J 2002 Electrokinetic microactuator arrays for active sublayer control of turbulent boundary layers $P h D$ Thesis University of Michigan, Ann Arbor, MI

[21] White F M 1991 Viscous Fluid Flow (New York: McGraw-Hill)

[22] Kline S J, Reynolds W C, Schraub F A and Runstadler P W 1967 The structure of turbulent boundary layers J. Fluid Mech. 30 741-73

[23] Kim H T, Kline S J and Reynolds W C 1971 The production of turbulence near a smooth wall in a turbulent boundary layer J. Fluid Mech. 50 133-68
[24] Robinson S K 1991 Coherent motion in the turbulent boundary layer Annu. Rev. Fluid Mech. 23 601-39

[25] Diez F J and Dahm W J A 2003 Electrokinetic microactuator arrays and system architecture for active sublayer control of turbulent boundary layers AIAA J. 41 1906-15

[26] Potter E C 1961 Electrochemistry, Principles and Applications (London: Cleaver-Hume)

[27] Burgreen D and Nakache F R 1964 Electrokinetic flow in ultrafine capillary slits J. Phys. Chem. 68 1084-91

[28] Reuss F F 1809 Sur un nouvel effet de l'éctricité galvanique Memoires de la Societé Imperiale de Naturalistes de Moscou 2 327-37

29] Rice C L and Whitehead R 1965 Electrokinetic flow in a narrow cylindrical capillary J. Phys. Chem. 69 4017-23

[30] Paul P H, Arnold D W and Rakestraw D J 1998 Electrokinetic generation of high pressures using porous microstructures $\mu$-TAS 98 Banff, Canada

[31] Gang W, Yang L, He Y, Zeng R, Cervera M L and Guardia M de la 2000 Mechanism of porous core electroosmotic pump flow injection system and its application to determination of chromium(VI) in waste-water Talanta 51 667-75

[32] Peters E C, Petro M, Svec F and Fréchet J M J 1998 Molded rigid polymer monoliths as separation media for capillary electrochromatography: 1. Fine control of porous properties and surface chemistry Anal. Chem. $702288-95$

[33] Peters E C, Petro M, Svec F and Fréchet J M J 1998 Molded rigid polymer monoliths as separation media for capillary electrochromatography: 2. Effect of chromatographic conditions on the separation Anal. Chem. 70 2296-2302 